\title{
Adubação fosfatada e níveis críticos de fósforo no solo para manutenção da produtividade do capim-elefante (Pennisetum purpureum cv. Napier) ${ }^{1}$
}

\section{Luciano de Melo Moreira ${ }^{2}$, Dilermando Miranda da Fonseca ${ }^{3}$, Janaina Azevedo Martuscello ${ }^{4}$, Elcivan Bento da Nóbrega ${ }^{5}$}

\author{
1 Parte da dissertação apresentada à UFV pelo quarto autor como parte das exigências para obtenção do título "Magister Scientiae". \\ 2 FEAD - Minas. Av. Brasil 1879 - Funcionários, Belo Horizonte, MG. \\ ${ }^{3}$ Departamento de Zootecnia da UFV - Viçosa, MG. \\ ${ }^{4}$ Doutorando do Departamento de Zootecnia da UFV - Viçosa, MG. \\ ${ }^{5}$ Departamento de Zootecnia da UNITINS - Araguaína, TO
}

RESUMO - Avaliou-se o efeito residual da adubação fosfatada de implantação sobre a produção de MS e os valores de níveis críticos de fósforo no solo para manutenção do capim-elefante (Pennisetum purpureum Schum. cv. Napier). Os tratamentos foram arranjados segundo fatorial $2 \times 2 \times 5$, em blocos casualizados com três repetições, e consistiram de duas formas de aplicação de fósforo (localizada no fundo do sulco e distribuída no sulco com incorporação), dois espaçamentos entre linhas de plantio $(0,5$ e $1,0 \mathrm{~m})$ e cinco doses de fósforo $(0,30,60,100$ e $150 \mathrm{~kg} / \mathrm{ha}$ de $\mathrm{P}$ e a $0,60,120,200$ e $300 \mathrm{~kg} / \mathrm{ha}, \mathrm{para}$ as aplicações localizada e distribuída, respectivamente). Foram efetuadas sete colheitas (cortes) das plantas nos sulcos (linhas) centrais da parcela, correspondente a $2 \mathrm{~m}^{2}$. Após pesagem da forragem colhida, amostras foram retiradas para determinação dos teores de MS. Amostras de solo também foram colhidas sobre as linhas de plantas após cada corte, para determinação dos teores de fósforo disponível pelo extrator Mehlich-1. Após cada corte, foi feita adubação em cobertura com nitrogênio e potássio $\left(67 \mathrm{~kg} /\right.$ ha de $\mathrm{N}$ e $\left.\mathrm{K}_{2} \mathrm{O}\right)$ utilizando-se sulfato de amônio e cloreto de potássio. A adubação fosfatada apresentou efeitos residuais sobre a produção de MS do capim-elefante durante dois anos após o estabelecimento da forrageira. Maiores produções foram verificadas no espaçamento de $0,5 \mathrm{~m}$ entre linhas de plantio. A forma de aplicação do fertilizante não influenciou o rendimento de MS. Os níveis críticos de fósforo no solo mostraram-se estáveis com a sucessão de cortes, apresentando maiores valores para o espaçamento de $1,0 \mathrm{~m}$ e na forma distribuída e incorporada no sulco para os dois espaçamentos.

Palavras-chave: espaçamento, extrator, forma de aplicação de fósforo, forrageira, matéria seca

\section{Phosphorus fertilization and soil phosphorus critical levels for the maintenance of elephantgrass (Pennisetum purpureum cv. Napier) crop productivity}

\begin{abstract}
The experiment was conducted over two years to evaluate the residual effect of phosphate fertilizer application on the DM yield and the soil P critical levels for maintenance of elephantgrass (Pennisetum purpureum Schum. cv. Napier) crop. The experimental treatments were arranged according to a $2 \times 2 \times 5$ factorial treatment combination of: two forms of $\mathrm{P}$ application (located on the bottom of the furrow or mixed with the soil in the furrow), two spacing of the planting lines $(0.5$ and $1.0 \mathrm{~m})$ and five phosphorus levels. The phosphorus levels were: $0,30,60100$ and $150 \mathrm{~kg} / \mathrm{ha}$ and $0,60,120,200$ and $300 \mathrm{~kg} / \mathrm{ha}$ of $\mathrm{P}$ for the located and mixed application, respectively. The experimental design was a randomized complete blocks one, with three replications. Seven harvests were taken in the central lines of the plots, corresponding to $2 \mathrm{~m}^{2}$. After weighing the harvested forage, samples were taken for DM contents determination. Soil samples were also collected along the planting lines after each harvest to establish phosphorus contents with Mehlich-1 extractor. After each harvest nitrogen and potassium fertilizers were broadcasted $\left(67 \mathrm{~kg} / \mathrm{ha}\right.$ of $\mathrm{N}$ and $\mathrm{K}_{2} \mathrm{O}$, as ammonium sulphate and potassium chloride, respectively). There was a residual phosphate fertilization effect on the elephantgrass dry matter yield over the two years experimental period. Plots planted at $0.5 \mathrm{~m}$ spacing reached higher forage yield. The fertilizer form of application did not influence forage yield. Soil phosphorus critical levels did not vary with harvesting sequence and reached higher values in plots planted to $1.0 \mathrm{~m}$ spacing and in those where the $\mathrm{P}$ fertilizer had been mixed with soil in the furrow, regardless of the spacing of planting.
\end{abstract}

Key Words: dry matter, forage, phosphorus application form, spacing

\section{Introdução}

A exploração da pecuária bovina brasileira, em geral, é baseada na utilização de pastagens. Entretanto, na maioria desses sistemas de produção, verificam-se baixos índices de produtividade das forrageiras, como conseqüência de vários fatores, destacando-se o manejo inadequado da atividade de pastejo e a baixa 
fertilidade do solo, os quais influenciam diretamente na sustentabilidade do sistema.

No Brasil, geralmente as pastagens são cultivadas em solos de baixa fertilidade, com generalizada deficiência e capacidade de adsorção de fósforo, além de elevados níveis de acidez. O fósforo, depois da água e do nitrogênio (moduladores da produtividade e persistência da pastagem), é o nutriente mais limitante à produção das plantas forrageiras, sendo de fundamental importância, principalmente no estabelecimento, estimulando a formação e o crescimento das raízes e o perfilhamento (Werner, 1986).

Entre as diversas espécies forrageiras utilizadas em sistemas intensivos de exploração da pecuária bovina, o capim-elefante (Pennisetum purpureum Schum. cv. Napier) é reconhecidamente de alto potencial produtivo e também uma das que mais respondem à adubação e, portanto, sua utilização pode ser recomendada para corte ou pastejo.

Nesse sentido, o conhecimento dos níveis críticos de fósforo no solo torna-se de alta relevância, por permitir a recomendação dos nutrientes na dose adequada para o crescimento inicial das plantas, de acordo com seus requerimentos. Por outro lado, os níveis críticos apresentam grandes variações entre espécies, tipos de solo, idade das plantas, forma de aplicação e de amostragem do solo, espaçamento entre plantas, entre outras. Quanto à idade da planta, tem-se verificado em várias espécies forrageiras decréscimo nos níveis críticos de fósforo com o crescimento e desenvolvimento da planta, o que evidencia menores exigências de fósforo, após o estabelecimento, ao longo do tempo (Fonseca et al., 1988; Guss et al., 1990a, 1990b; Santos et al., 2002).

Em razão da contínua absorção de fósforo pelas plantas, aliada à complexidade desse nutriente nos solos, questiona-se por quanto tempo a concentração adequada ao crescimento inicial é suficiente para manter determinada produtividade da forrageira. Tem-se observado freqüentemente, sob regime de corte ou pastejo, real degradação das pastagens após 4 a 5 anos de utilização, causada provavelmente pelo decréscimo dos teores de fósforo disponível no solo, podendo influenciar também na absorção de nitrogênio e/ou de potássio.

Apesar da reconhecida importância da adubação fosfatada por ocasião do estabelecimento da pastagem, são escassos os trabalhos na literatura que evidenciam seu efeito residual sobre as produções dos anos subseqüentes. Portanto, é fundamental o desenvolvimento de trabalhos visando à determinação dos níveis críticos de fósforo no solo para manutenção da produtividade desejada ao longo dos anos, garantindo maior longevidade da pastagem.
Objetivou-se avaliar o efeito residual da adubação fosfatada de implantação sobre a produção de MS, bem como estimar os níveis críticos de fósforo no solo para manutenção da produtividade do capim-elefante cv. Napier sob regime de corte.

\section{Material e Métodos}

O estudo foi conduzido no Campo Agrostológico do Departamento de Zootecnia da Universidade Federal de Viçosa, MG, em continuidade ao trabalho de Ruppin (1997), que estimou os níveis críticos de fósforo para o estabelecimento do capim-elefante (Pennisetum purpureum Schum. cv. Napier). O experimento foi instalado em 18 de dezembro de 1995, em um Latossolo Vermelho-Amarelo de textura argilosa e topografia ondulada.

O clima da região é do tipo Cwa, segundo classificação de Köppen (1948), com precipitação anual em torno de $1.340 \mathrm{~mm}$, estiagem no período de maio a setembro e temperaturas médias anuais máximas e mínimas de 22,0 e $15,0^{\circ} \mathrm{C}$, respectivamente.

As características químicas e físicas do solo na camada de 0 a $20 \mathrm{~cm}$ de profundidade, antes do plantio do capimelefante, foram: $\mathrm{pH}=5,5\left(\mathrm{H}_{2} \mathrm{O}, 1: 2,5\right) ; \mathrm{PeK}$ (Mehlich-1) =0,6 e $16 \mathrm{mg} / \mathrm{dm}^{3}$, respectivamente; $\mathrm{Ca}, \mathrm{Mg}$ e $\mathrm{Al}(\mathrm{KCl} 1 \mathrm{~mol} / \mathrm{L})=$ 1,$4 ; 0,3 \mathrm{e} 0,2 \mathrm{cmol}_{\mathrm{c}} / \mathrm{dm}^{3}$, respectivamente; argila, silte, areia fina, areia grossa $=56,5,15$ e $24 \%$, respectivamente.

O delineamento experimental foi em blocos casualizados com três repetições, em um esquema fatorial 2 × 2 × 5 , em que os fatores consistiram de duas formas de aplicação de $\mathrm{P}$ : localizado no fundo do sulco (PL) e distribuído ao longo da superfície do sulco (PD) com posterior incorporação por meio de uma enxada, de forma que reagisse com certo volume de solo da camada superficial; dois espaçamentos entre as linhas de plantio $(0,5$ e $1,0 \mathrm{~m})$ e cinco doses de $\mathrm{P}$ $(0 ; 1 ; 2 ; 3,3$ e 5$)$.

As doses corresponderam, respectivamente, a 0,30,60, 100 e $150 \mathrm{~kg} / \mathrm{ha}$, na aplicação localizada, e a 0, 60, 120, 200 e $300 \mathrm{~kg} / \mathrm{ha}$, na aplicação distribuída. Nas parcelas de $16 \mathrm{~m}^{2}$ (4 x $4 \mathrm{~m}$ ) com quatro ou oito linhas, dependendo do espaçamento, aplicou-se o adubo correspondente às doses de $\mathrm{P}$ no momento do plantio.

$\mathrm{Na}$ fase de implantação (estabelecimento) empregou-se o manejo descrito por Ruppin (1997). As avaliações dos efeitos residuais da aplicação de fósforo no capim-elefante tiveram início após a segunda colheita (corte), abrangendo o período de abril/96 a maio/98. Foram efetuados sete cortes, de modo que a produção dos cortes 1 e 4 correspondeu ao período de crescimento da forrageira na época seca do ano, 
e a dos demais, a intervalos de aproximadamente 60 dias, com exceção do corte 7, que foi de 75 dias, durante o período chuvoso. Os dados referentes às condições climáticas e aos dias de intervalos de cortes durante todo o período experimental são apresentados na Tabela 1 .
As avaliações foram feitas após colheita das plantas a $2 \mathrm{~cm}$ do nível do solo, constantes nas duas e quatro linhas centrais da unidade experimental (área útil de $2 \mathrm{~m}^{2}$ ), correspondentes, respectivamente, aos espaçamentos de 1,0 e $0,5 \mathrm{~m}$.

Tabela 1 - Intervalo de cortes, precipitação pluvial total $(\mathrm{mm})$, temperatura média diária $\left({ }^{\circ} \mathrm{C}\right)$ e insolação média diária (h) no período de abril/96 a maio/98

Table 1 - Cut interval, monthly rainfall, temperature and heatstroke from April/96 to May/98

\begin{tabular}{|c|c|c|c|c|c|c|c|}
\hline $\begin{array}{l}\text { Característica } \\
\text { Characteristc }\end{array}$ & $\begin{array}{c}\text { Corte } 1^{1} \\
\text { Cut } 1\end{array}$ & $\begin{array}{c}\text { Corte } 2 \\
\text { Cut } 2\end{array}$ & $\begin{array}{c}\text { Corte } 3 \\
\text { Cut } 3\end{array}$ & $\begin{array}{c}\text { Corte } 4 \\
\text { Cut } 4\end{array}$ & $\begin{array}{c}\text { Corte } 5 \\
\text { Cut } 5\end{array}$ & $\begin{array}{c}\text { Corte } 6 \\
\text { Cut } 6\end{array}$ & $\begin{array}{c}\text { Corte } 7 \\
\text { Cut } 7\end{array}$ \\
\hline $\begin{array}{l}\text { Intervalo } \\
\text { Interval }\end{array}$ & 238 & 61 & 63 & 184 & 64 & 62 & 75 \\
\hline $\begin{array}{l}\text { Precipitação } \\
\text { Rainfall }\end{array}$ & 572,9 & 569,6 & 218,0 & 174,5 & 432,0 & 356,8 & 171,8 \\
\hline $\begin{array}{l}\text { Temperatura } \\
\text { Temperature }\end{array}$ & 19,48 & 23,65 & 22,31 & 19,57 & 23,59 & 25,20 & 23,30 \\
\hline $\begin{array}{l}\text { Insolação } \\
\text { Heatstroke }\end{array}$ & 5,98 & 5,58 & 5,99 & 7,25 & 4,82 & 6,93 & 6,57 \\
\hline
\end{tabular}

${ }^{1}$ Cortes 1, 2, 3, 4, 5, 6 e 7, efetuados respectivamente em 11/12/96, 10/02/97, 14/04/97, 14/10/97, 16/12/97, 16/02/98, 29/04/98.

2 Número de dias de crescimento que antecederam ao respectivo corte.

Fonte: Estação meteorológica da UFV.

1 Cuts 1, 2, 3, 4, 5, 6, and 7 made respectively on 12/11/96, 02/10/97, 04/14/97, 10/14/97, 12/16/97, 05/16/98, and 04/29/98.

2 Number of days of growth that preceeded the respective cut.

Source: Meteorological Station of UFV.

Após o corte e a pesagem da forragem, amostras foram retiradas, fracionadas em colmos (picados em pedaços de 3 a $5 \mathrm{~cm}$ ) e lâminas foliares e, em seguida, pesadas e colocadas em estufa de circulação forçada a $65^{\circ} \mathrm{C}$ até peso constante. Depois de secas, as amostras foram pesadas, moídas e acondicionadas em frascos de vidro para posteriores análises laboratoriais e estimativas de produção de MS (kg/ha), segundo procedimentos descritos por Silva \& Queiroz(2002).

Efetuaram-se, após cada corte, adubações com nitrogênio e potássio nas doses de $67 \mathrm{~kg} / \mathrm{ha}$ de $\mathrm{N}$ e de $\mathrm{K}_{2} \mathrm{O}$, empregando-se as fontes sulfato de amônio e cloreto de potássio, respectivamente, e distribuindo-se a mistura dos dois fertilizantes entre as linhas das plantas, incorporando-a e simultaneamente eliminando as plantas daninhas presentes na área.

As amostragens para avaliação dos teores de fósforo disponível no solo foram feitas após os cortes $(1,3,5$ e 7$)$, com um trado tipo holandês, à profundidade de 0 a $20 \mathrm{~cm}$. As amostragens foram feitas somente nestes cortes, para se evitar maiores perturbações nas parcelas, haja vista seu tamanho reduzido, retirando-se grande número de amostras por parcelas (12 e 6 subamostras de solo por parcela nos espaçamentos de 0,5 e 1,0 m entre linhas, respectivamente, alocadas sobre as linhas de plantas recém-colhidas na área útil da parcela). Cada conjunto de subamostras, depois de homogeneizadas, deu origem a uma amostra composta na qual foram determinados os teores de fósforo disponível pelo extrator Mehlich-1 (Braga \& Defelipo, 1974).

Equações de regressão foram estimadas para os dados (média de três repetições) de produção de MS da parte aérea, em função das doses de fósforo aplicadas no plantio, para cada espaçamento e cada forma de aplicação do fertilizante fosfatado. A partir dessas equações, foram estimadas as doses recomendáveis de fósforo para obtenção de $90 \%$ da produção máxima. Quando a dose de fósforo estimada por derivação para produção máxima extrapolou o espaço experimental, considerou-se $90 \%$ da produção na maior dose testada.

Foram também ajustadas equações de regressão entre os teores de fósforo disponível no solo pelo extrator Mehlich-1 conforme as doses de fósforo aplicadas no plantio, referentes aos cortes 1, 3, 5 e 7. Substituindo nessas equações a dose recomendável de fósforo relativa a $90 \%$ da produção máxima, estimaram-se os níveis críticos de fósforo no solo para manutenção do capim-elefante nos diferentes espaçamentos e nasformas de aplicação do fertilizante fosfatado.

Para a seleção das equações de regressão, foram considerados os modelos com os maiores coeficientes de determinação $\left(\mathrm{R}^{2}\right)$, independentemente da significância dos coeficientes angulares das equações, utilizando-se o programa SAEG - Sistema de análises estatísticas e genéticas (Euclides, 2003). 


\section{Resultados e Discussão}

A produção de MS da parte aérea do capim-elefante adubado com P no plantio variou com o espaçamento entre linhas na maioria dos cortes seguintes aos da fase de estabelecimento (Tabela 2). As maiores produções foram verificadas no espaçamento de $0,5 \mathrm{~m}$, com diferenças significativas ( $\mathrm{P}<0,01)$ em relação ao espaçamento de $1,0 \mathrm{~m}$ na maioria dos cortes. Esse efeito de maiores produções no menor espaçamento pode ser atribuído à sua maior população de plantas.

Por outro lado, com exceção dos dados de produção no corte 3 , não foi detectada influência $(\mathrm{P}>0,05)$ da forma de aplicação do adubo fosfatado sobre as produções de MS, embora os valores em todos os cortes tenham sido sempre maiores, independentemente do espaçamento, quando a adubação foi distribuída no sulco de plantio (PD). Resultados semelhantes foram reportados por Novais et al. (1985) e Fonseca et al. (1997a), que observaram maiores rendimentos forrageiros quando o fertilizante foi aplicado em todo ou em parte do volume do solo, condição que possibilita maior contato das raízes com o nutriente.

Os dados de produção de MS, conforme as doses de fósforo aplicadas no plantio, foram ajustados a modelos quadráticos e apresentaram elevados valores dos coeficientes de determinação $\left(\mathrm{R}^{2}\right)$ (Tabela 3 ). Este resultado indica que houve efeito residual das doses de $\mathrm{P}$ aplicadas por ocasião do plantio e comprova, pela significância dos coeficientes angulares na maioria das equações, com a sucessão dos cortes, que a produção de MS se manteve praticamente constante quando as condições climáticas foram favoráveis.

A manutenção da produtividade com a sucessão dos cortes é indício de que as doses de fósforo foram suficientes para manter a concentração de fósforo necessária para obtenção de elevadas produções, sem necessidade de aplicações adicionais. A maioria dos valores dos coeficientes de regressão linear das equações relacionando fósforo disponível no solo e doses do nutriente (Tabela 6) foi muito semelhante nos sete cortes, o que reforça as evidências do efeito residual das doses de fósforo aplicadas no plantio.

As produções de MS relativas a $90 \%$ da produção máxima, bem como nas maiores doses de fósforo aplicadas, são descritas na Tabela 4. As produções, na maioria dos cortes, exceto no corte 4 , foram elevadas, provavelmente em decorrência da não-limitação dos fatores de ambiente, como luz, água e temperatura (Tabela 1), e dos efeitos das adubações com nitrogênio e potássio. Ao considerar a produção dos sete cortes no intervalo de 65 dias, obteve-se taxa de produção de $224 \mathrm{~kg} /$ ha.dia de MS.
A variação nos valores das doses recomendáveis de P com a sucessão de cortes (Tabela 4) pode ser atribuída a variações de fatores de ambiente durante o período experimental (período chuvoso, de transição e seco), influenciando as produções de MS. Os elevados valores das doses estimadas para a forma distribuída do nutriente (PD) (Tabela 4) provavelmente resultaram do decréscimo nas reações de solubilização do fosfato no solo do respectivo tratamento.

As doses recomendáveis de fósforo para a manutenção da produtividade, em ambos os tratamentos PL e PD (Tabela 4), foram superiores às estimativas encontradas por Saraiva \& Carvalho (1991) para o estabelecimento do capimelefante. Esses autores registraram valores de aproximadamente $49,0 \mathrm{~kg} / \mathrm{ha}$ de $\mathrm{P}$, com níveis críticos no solo em torno de $7 \mathrm{mg} / \mathrm{dm}^{3}$, e atribuíram esse baixo valor à aplicação localizada do fósforo e ao método de amostragem do solo. Nesse contexto, Fonseca et al. (1997a,b) também constataram que esses dois fatores foram os que mais influenciaram os valores de níveis críticos.

Ruppin (1997) verificou que a produção é mais bem explicada pelos teores de fósforo disponível na linha de plantio, pois apresentaram melhor correlação com a produção de MS da parte aérea do capim-elefante. Da mesma forma, as doses recomendáveis de fósforo correspondentes a $90 \%$ da produção máxima - e que também, espera-se, são as de máxima eficiência econômica (Tabela 4) - foram bem superiores às reportadas por Werner (1986), que registrou valor de $21,8 \mathrm{~kg} / \mathrm{ha}$ de $\mathrm{P}$ para adubação fosfatada de manutenção em pastagem exclusiva de gramínea a ser aplicada somente quando os teores de fósforo no solo estiverem abaixo de $10 \mathrm{mg} / \mathrm{dm}^{3}$.

As baixas e menores produções de MS observadas no corte 4 (Tabela 4) se devem, possivelmente, ao período seco que antecedeu sua realização (Tabela 1), quando ocorreram, simultaneamente, déficit hídrico e baixas temperaturas, limitando drasticamente as produções. Embora no corte 7 também tenham ocorrido menores insolação e precipitação, o número de dias que antecedeu o corte foi maior, possibilitando acúmulo de biomassa. A limitação hídrica influencia diretamente o crescimento e desenvolvimento das plantas, retardando a divisão, e pode ser explicada pela diminuição da atividade da amilase, translocação de amido dos cloroplastos, ocasionando redução da atividade fotossintética da planta (Gomide, 1994). A aplicação do fósforo distribuída ao longo da superfície do sulco (PD) e o espaçamento de 1,0 m entre linhas de plantas influenciaram $(\mathrm{P}<0,01)$ os teores de fósforo disponível no solo em todos os cortes avaliados (Tabela 5). Os mais altos teores de fósforo na aplicação distribuída e 
Tabela 2 - Produção de MS da parte aérea das plantas de capim-elefante, conforme a forma de aplicação do fertilizante fosfatado, o espaçamento entre linhas de plantio e os níveis de fósforo nos sete cortes

Table 2 - Elephantgrass shoot DM yield according to the form of $P$ application, the spacing of the planting lines and the $P$ levels in the seven cuts

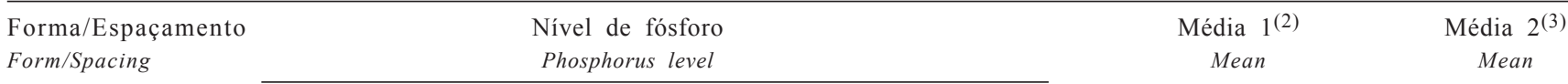

Form/Spacing

$\begin{array}{lllll}0 & 1(1) & 2 & 3,3 & 5\end{array}$

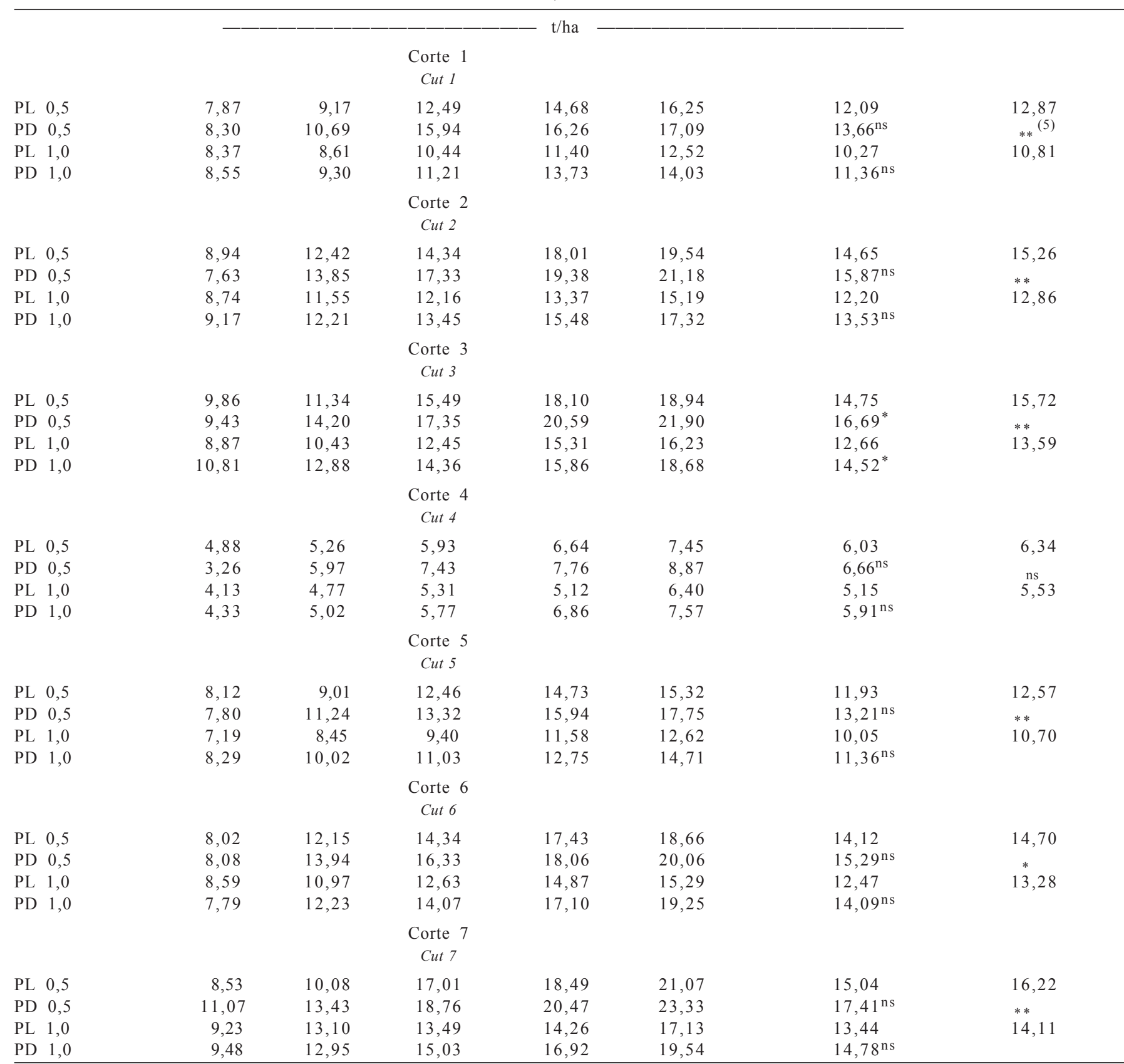

PL; PD: Fertilizante fosfatado localizado no fundo do sulco e distribuído e incorporado no sulco, respectivamente; 0,5; 1,0: Espaçamento de 0,5 e 1,0 m, respectivamente, entre as linhas de plantio.

PL; PD: Phosphate fertilizer localized at the holes bottom and distributed and incorporated in the holes, respectively; 0,5; 1,0: Spacing of 0.5 and 1.0 m between the plantation lines, respectively.

${ }_{1}^{1}$ Nível 1 correspondente à dose de 30 e $60 \mathrm{~kg} / \mathrm{ha}$ de $P$ para $P L$ e PD, respectivamente.

1 Level 1 corresponding to the 30 and $60 \mathrm{~kg} / \mathrm{ha} P$ dose to $P L$ and $P D$, respectively.

2 Média por forma de aplicação e por espaçamento; ${ }^{3}$ Média por espaçamento; ${ }^{4}$ Compara PL vs PD dentro do mesmo espaçamento; ${ }^{5}$ Compara espaçamento $0,5 \mathrm{~m}$ vs espaçamento $1,0 \mathrm{~m}$.

${ }_{2}^{2}$ Average for the $P$ application form and for the spacing; ${ }^{3}$ Average for spacing; ${ }^{4}$ Compares $P L$ vs PD in the same spacing. ${ }^{5}$ Compares the $0.5 \mathrm{~m}$ spacing vs the $1.0 \mathrm{~m}$ spacing

ns, ${ }^{*},{ }^{* *}$ : Não-significativo, significativo a 5 e $1 \%$, respectivamente.

Ns, ${ }^{*}, * *$ : Not significant, significant at 5 and $1 \%$, respectively.

O efeito não-significativo da forma de aplicação do fertilizante sobre a produção de MS (Tabela 2) na maioria dos cortes pode ser atribuído ao pequeno volume de solo onde o fertilizante foi incorporado, ou seja, houve tendência de a distribuição do adubo ser semelhante à da forma localizada, o que provavelmente resultou em igual distribuição do sistema radicular. 
Tabela 3 - Produção de matéria seca (MS) da parte aérea das plantas de capim-elefante (t/ha), de acordo com as doses de fósforo adicionadas no plantio, em $\mathrm{kg} / \mathrm{ha}$ de $\mathrm{P}$, conforme a forma de aplicação do fertilizante e o espaçamento entre linhas de plantio nos sete cortes

Table 3 - Elephantgrass shoot DM yield ( $t / h a)$ as affected by the $P$ levels added in the establishing, in $\mathrm{kg} / \mathrm{ha}$ of $P$, according to the form of $P$ application and the spacing of the planting lines in the seven cuts

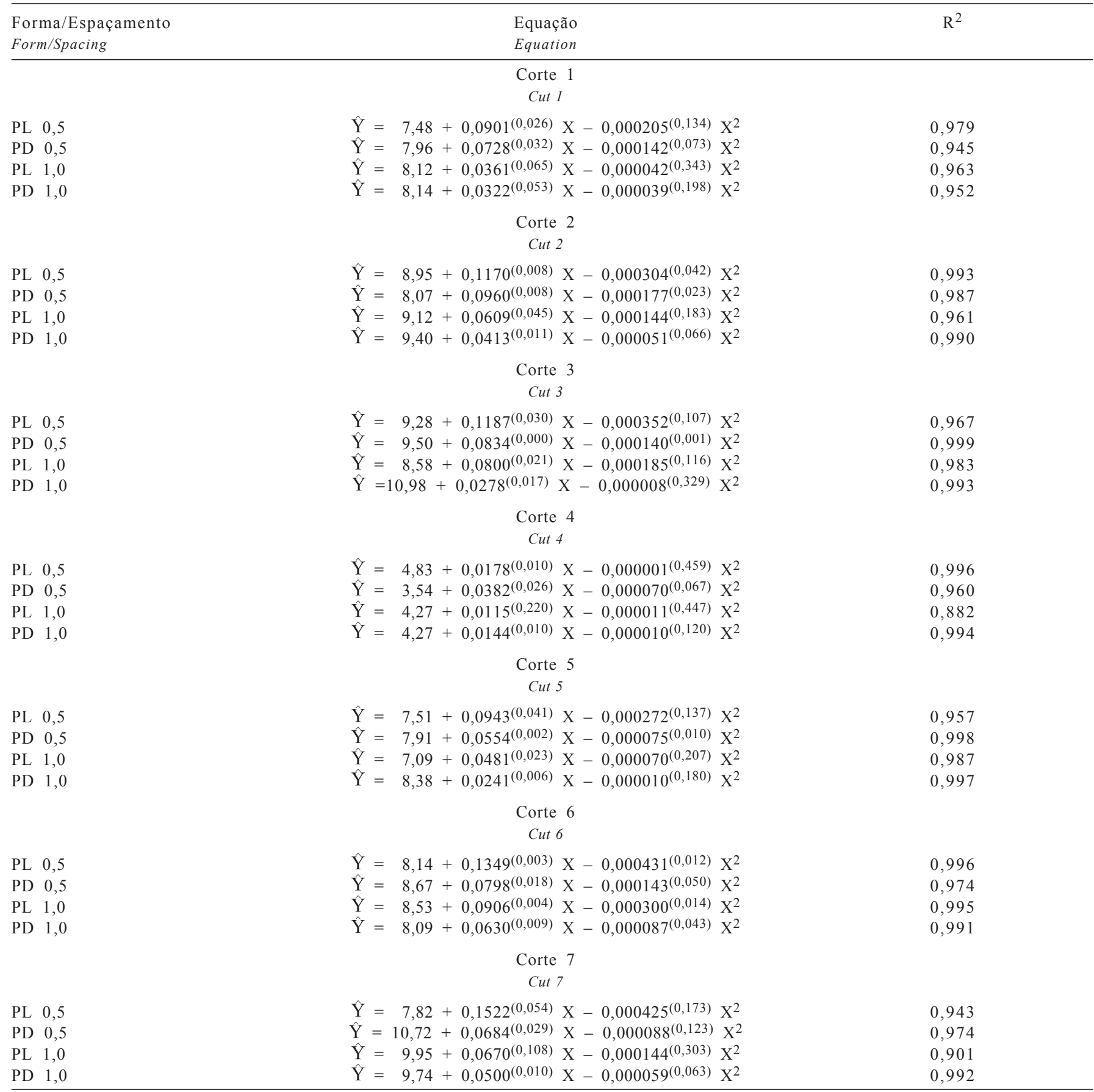

PL; PD: Fertilizante fosfatado localizado no fundo do sulco e distribuído e incorporado no sulco, respectivamente.

0,$5 ; 1,0$ : Espaçamento de 0,5 e 1,0 m entre as linhas de plantio, respectivamente.

$P L ; P D:$ Phosphorus fertilizer located on the botton of the furrow or mixed with the soil in the furrow, respectively.

0,$5 ; 1,0$ : Spacing of 0.5 and $1.0 \mathrm{~m}$ between the planting lines, respectively. 
Tabela 4 - Produção de MS da parte aérea das plantas de capim-elefante, correspondente a $90 \%$ da produção máxima, e doses recomendáveis de fósforo, conforme a forma de aplicação do fertilizante fosfatado e o espaçamento entre linhas de plantio nos sete cortes

Table 4 - Elephantgrass shoot DM yield, corresponding to $90 \%$ of the maximum production, and P recommended levels according to the form of $P$ application and the spacing of the planting lines in the seven cuts

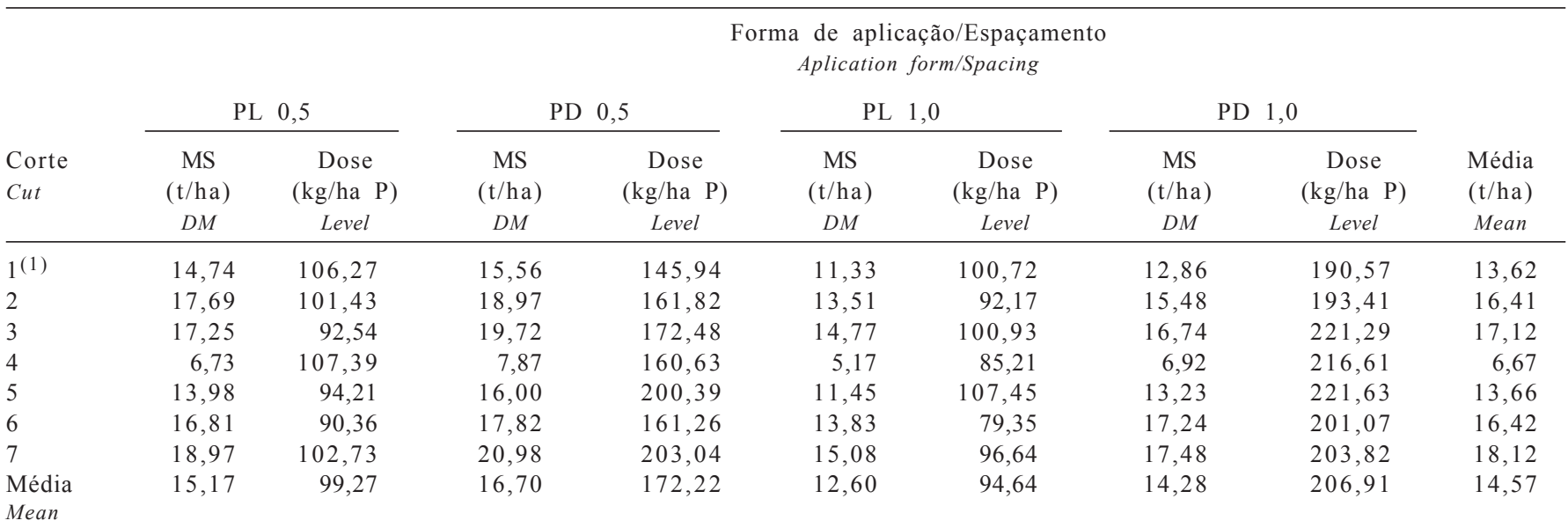

PL; PD: Fertilizante fosfatado localizado no fundo do sulco e distribuído e incorporado no sulco, respectivamente.

0,$5 ; 1,0$ : Espaçamento de 0,5 e 1,0 m entre as linhas de plantio, respectivamente.

$P L ; P D:$ Phosphorus fertilizer located on the botton of the furrow or mixed with the soil in the furrow, respectively.

0,$5 ; 1,0$ : Spacing of 0.5 and $1.0 \mathrm{~m}$ between the planting lines, respectively.

incorporada (PD) decorreram, provavelmente, das maiores doses aplicadas nessa modalidade e da forma de amostragem do solo (efetuada sobre a linha de plantio), o que proporcionou maior probabilidade de o trado atingir a zona fertilizada na forma distribuída (PD) que na forma localizada (PL). Os maiores teores de fósforo disponível $(\mathrm{P}<0,01)$ no espaçamento de $1,0 \mathrm{~m}$, comparativamente aos do espaçamento de $0,5 \mathrm{~m}$, em todos os cortes, podem ser explicados pelo fato de no maior espaçamento ter ocorrido maior concentração do nutriente, por ter sido considerada a mesma quantidade por área.

Para as duas formas de aplicação do nutriente e nos dois espaçamentos entre as linhas de plantio, relativos aos cortes 1, 3, 5 e 7 (Tabela 7), observou-se, de modo geral, que o valor de nível crítico na forma distribuída foi $45 \%$ superior ao da forma localizada. Esses maiores valores para o fósforo distribuído ao longo da superfície (PD) não eram esperados, mas podem ser atribuídos ao fato de as doses terem sido duas vezes às da forma localizada (PL), além da provável influência, conforme já mencionado anteriormente, das amostragens de solo após cada corte (colheita) do capimelefante. Resultado semelhante (43\%) foi obtido por Fonseca et al. (1997b), em solo de textura argilosa, para fase de estabelecimento do capim-colonião. Esses autores verificaram que o método de aplicação do fertilizante e a forma de amostragem do solo foram os fatores que mais influenciaram os níveis críticos de fósforo no solo, mesmo quando outras variáveis (solo, espécie, extrator, idade da planta) foram mantidas constantes.
Em geral, não houve variação acentuada dos valores de níveis críticos dentro de cada forma de aplicação do fertilizante e espaçamento entre linhas de plantio (Tabela 7), com a sucessão de cortes, o que evidencia estabilização dos valores de níveis críticos. A não-redução dos níveis críticos com a sucessão de cortes contraria os resultados descritos por Santos et al. (2002), que estudaram as gramíneas Brachiaria decumnbens cv. Basilisk e Panicum maximum cv. Mombaça e verificaram decréscimo em níveis críticos com a idade das plantas $(14,28,42,56$ e 70 dias pós-emergência).

Considerando-se que não foi detectado efeito $(\mathrm{P}>0,05)$ das formas de aplicação do fertilizante sobre as produções de MS, as médias dos valores de níveis críticos no solo foram 26 e $53 \mathrm{mg} / \mathrm{dm}^{3}$, para os espaçamentos de 0,5 e $1,0 \mathrm{~m}$ entre as linhas de plantio, respectivamente, necessários para a manutenção da produtividade do capim-elefante por, aproximadamente, dois anos após seu estabelecimento.

Os valores de níveis críticos para a manutenção foram inferiores aos estimados para o estabelecimento do capimelefante na mesma área experimental (Ruppin, 1997). Ao considerar os resultados do corte 7, realizado aproximadamente dois anos após a fase de estabelecimento, os valores de níveis críticos de manutenção de 27 e $45 \mathrm{mg} / \mathrm{dm}^{3}$ (média do espaçamento de 0,5 e 1,0 m, respectivamente, independentemente da forma de aplicação) foram 82 e $48 \%$ dos valores para o estabelecimento da gramínea (Ruppin, 1997) nos espaçamentos de 0,5 e 1,0 m, respectivamente. Esses resultados confirmam as evidências e constatações de outros autores sobre a queda dos valores de níveis críticos 
Tabela 5 - Teores de fósforo $\left(\mathrm{mg} / \mathrm{dm}^{3}\right.$ ) disponível no solo (amostragem sobre as linhas de plantio na profundidade de 0 a $20 \mathrm{~cm}$ ), conforme a forma de aplicação do fertilizante fosfatado, o espaçamento entre linhas de plantio e o nível de fósforo, relativos a quatro cortes

Table 5 - Phosphorus rates ( $\mathrm{mg} / \mathrm{dm}^{3}$ ) available in the soil (plantating lines sampling at 0 to $20 \mathrm{~cm}$ depth) according to the form of $P$ application, the spacing of the planting lines and the $P$ levels in the four cuts

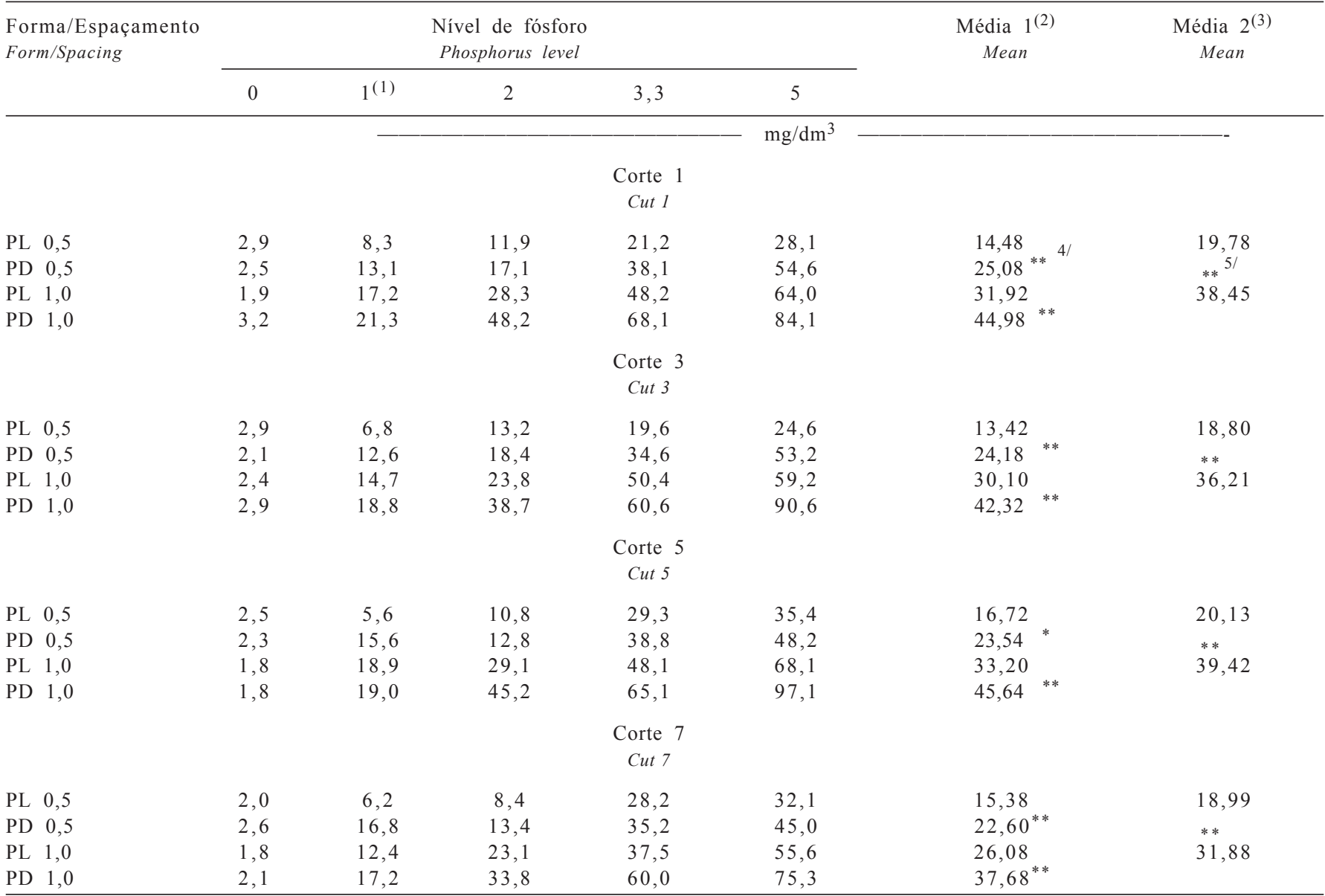

PL; PD: Fertilizante fosfatado localizado no fundo do sulco e distribuído e incorporado no sulco, respectivamente.

0,$5 ; 1,0$ : Espaçamento de 0,5 e $1,0 \mathrm{~m}$ entre as linhas de plantio, respectivamente.

${ }_{1}^{1}$ Nível 1 correspondente às doses de 30 e $60 \mathrm{~kg} / \mathrm{ha}$ de $\mathrm{P}$, para $\mathrm{PL}$ e PD, respectivamente.

2 Média por forma de aplicação e por espaçamento.

3 Média por espaçamento.

${ }^{4}$ Compara PL vs PD dentro do mesmo espaçamento.

5 Compara espaçamento $0,5 \mathrm{~m}$ vs espaçamento $1,0 \mathrm{~m}$

*, **: Significativo a 5 e $1 \%$, respectivamente.

$P L ; P D:$ Phosphorus fertilizer located on the botton of the furrow or mixed with the soil in the furrow, respectively.

0,$5 ; 1,0$ : Spacing of 0.5 and $1.0 \mathrm{~m}$ between the planting lines, respectively.

1 Level 1 corresponds to the levels of 30 and $60 \mathrm{~kg} / \mathrm{ha} P$ for $P L$ and PD, respectively.

2 Average for the application form and for the spacing.

3 Average for spacing.

${ }^{4}$ Compares $P L$ vs $P D$ in the same spacing.

5 Compares the $0.5 \mathrm{~m}$ spacing vs the $1.0 \mathrm{~m}$ spacing.

*,** : significant at 5 and $1 \%$, respectively.

após a fase de estabelecimento dos capins andropógon, braquiária e jaraguá (Fonseca et al., 1988), braquiárias e leguminosas (Guss et al., 1990a,b) e do capim-mombaça (Santos et al., 2002). O maior requerimento de fósforo pelas plantas forrageiras no plantio pode ser atribuído ao maior valor da constante de cinética de absorção de fósforo $(\mathrm{Km})$ nessa fase (Vale, 1982; Fonseca et al., 1997a).

Vale (1982), trabalhando com eucalipto e milho, constatou valores superiores da constante de cinética de absor- ção de fósforo na fase inicial de crescimento dos clones de eucalipto, diminuindo com o aumento da idade das plantas. Tynker, citado por Fonseca (1995), também atribuiu a redução dos valores de níveis críticos de fósforo no solo com o aumento da idade das plantas ao maior volume de solo explorado pelas plantas perenes, às possíveis associações micorrízicas favorecendo a absorção de fósforo e à diminuição da cinética de absorção de fósforo $(\mathrm{Km})$. 
Tabela 6 - Equações de regressão ajustadas aos teores de fósforo $\left(\mathrm{mg} / \mathrm{dm}^{3}\right)$ disponível no solo (amostragem sob as linhas de plantio na profundidade de 0 a $20 \mathrm{~cm}$ ), de acordo com as doses de fósforo adicionadas no estabelecimento, em kg/ha de $\mathrm{P}$, conforme a forma de aplicação do fertilizante fosfatado e o espaçamento entre linhas de plantio, relativas a quatro cortes

Table 6 - Adjusted regression equations on the phosphorus rates $\left(\mathrm{mg} / \mathrm{dm}^{3}\right)$ available in the soil (planting lines sampling at 0 to $20 \mathrm{~cm}$ depth) according to the form of $P$ application and the spacing of the planting lines in the four cuts

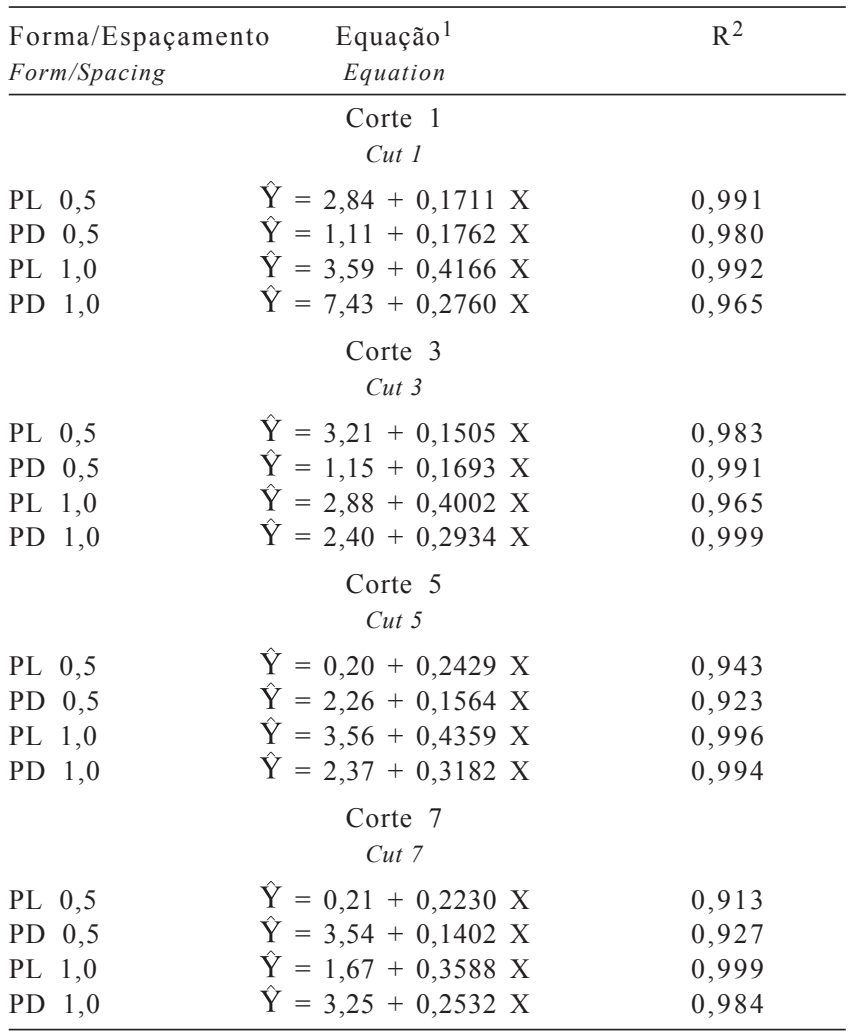

PL; PD: Fertilizante fosfatado localizado no fundo do sulco e distribuído e incorporado no sulco, respectivamente.

0,5 ; 1,0: Espaçamento de 0,5 e $1,0 \mathrm{~m}$ entre as linhas de plantio, respectivamente.

1 Todos os coeficientes foram significativos a $0,1 \%$ de probabilidade. $P L ; P D$ : Phosphorus fertilizer located on the botton of the furrow or mixed with the soil in the furrow, respectively.

0,5; 1,0: Spacing of 0.5 and $1.0 \mathrm{~m}$ between the planting lines, respectively.

${ }^{1}$ All the coefficients were significant at $0.1 \%$ probability level.

\section{Conclusões}

A adubação fosfatada possui efeitos residuais que proporcionam a manutenção da produção de MS do capimelefante durante dois anos após fase de estabelecimento da forrageira.

\section{Literatura Citada}

BRAGA, J.M.; DEFELIPO, B.V. Determinação espectrofotométrica do fósforo em extrato de solos e plantas. Revista Ceres, v.21, p.73-85, 1974.

EUCLIDES, R.F. Sistema de análise estatística e genética. Viçosa, MG: Universidade Federal de Viçosa, 2003. 68p.
Tabela 7 - Níveis críticos de fósforo no solo, pelo extrator Mehlick1, para a manutenção de $90 \%$ da produção máxima do capim-elefante, conforme a forma de aplicação do fertilizante fosfatado e o espaçamento entre linhas de plantio, relativos a quatro cortes após a fase de estabelecimento

Table 7 - Phosphorus critical levels in the soil, by the Mehlich-1 extractor, corresponding to $90 \%$ elephantgrass maximum production, according to the form of $P$ application and the spacing of the planting lines in the four cuts after establishing

\begin{tabular}{lccccc}
$\begin{array}{l}\text { Forma/Espaçamento } \\
\text { Form/Spacing }\end{array}$ & $\begin{array}{c}\text { Corte } 1 \\
\text { Cut } 1\end{array}$ & $\begin{array}{c}\text { Corte } 3 \\
\text { Cut } 3\end{array}$ & $\begin{array}{c}\text { Corte } 5 \\
\text { Cut } 5\end{array}$ & $\begin{array}{c}\text { Corte } 7 \\
\text { Cut } 7\end{array}$ & $\begin{array}{c}\text { Média } \\
\text { Mean }\end{array}$ \\
\hline & & & $\mathrm{mg} / \mathrm{dm}^{3}$ P & \\
PL 0,5 & 21 & 17 & 23 & 23 & 21 \\
PD 0,5 & 27 & 30 & 34 & 32 & 31 \\
PL 1,0 & 45 & 43 & 50 & 36 & 43 \\
PD 1,0 & 60 & 67 & 73 & 55 & 64
\end{tabular}

PL; PD: Fertilizante fosfatado localizado no fundo do sulco e distribuído $e$ incorporado no sulco, respectivamente.

0,5; 1,0: Espaçamento de 0,5 e $1,0 \mathrm{~m}$ entre as linhas de plantio, respectivamente.

PL; PD: Phosphorus fertilizer located on the botton of the furrow or mixed with the soil in the furrow, respectively.

0,$5 ; 1,0$ : Spacing of 0.5 and $1.0 \mathrm{~m}$ between the planting lines, respectively.

FONSECA, D.M.; ALVAREZ V., V.H.; NEVES, J.C.L. et al. Níveis críticos de fósforo em amostras de solos para o estabelecimento de Andropogon gayanus, Brachiaria decumbens e Hyparrhenia rufa. Revista Brasileira de Ciência do Solo, v.12, n.1, p.49-58, 1988.

FONSECA, D.M.; GOMIDE, J.A.; ALVAREZ V., V.H. et al. Fatores que influenciam os níveis críticos de fósforo para o estabelecimento de gramíneas forrageiras: I. Casa de vegetação. Revista Brasileira de Ciência do Solo, v.21, p.27-34, 1997 a.

FONSECA, D.M.; GOMIDE, J.A.; ALVAREZ V., V.H. et al. Fatores que influenciam os níveis críticos de fósforo para o estabelecimento de gramíneas forrageiras: II. Em campo. Revista Brasileira de Ciência do Solo, v.21, p.35-40, 1997 b.

GOMIDE, J.A. Fisiologia do crescimento livre de plantas forrageiras. In: PEIXOTO, A.M.; MOURA, J.C.; FARIA, V.P. (Eds). Pastagens: fundamentos da exploração racional. 2.ed. Piracicaba: Fundação de Estudos Agrários Luiz de Queiroz, 1994. p.1-14.

GUSS, A.; GOMIDE, J.A.; NOVAIS, R.F. Exigência de fósforo para o estabelecimento de quatro espécies de Brachiaria em solos com características físico-químicas distintas. Revista da Sociedade Brasileira de Zootecnia, v.19, n.4, p.278-289, $1990 \mathrm{a}$.

GUSS, A.; GOMIDE, J.A.; NOVAIS, R.F. Exigência de fósforo para o estabelecimento de quatro leguminosas forrageiras tropicais em solos com características físico-químicas distintas. Revista da Sociedade Brasileira de Zootecnia, v.19, n.5, p.450-458, 1990 b.

KÖPPEN, W. Climatologia. Trad. Pedro R.H. Perez. Buenos Aires, Panamericana, 1948. 478p.

MARSCHNER, H. Mineral nutrition of higher plants. 2.ed. New York: Academic Press, 1995. 874p.

NOVAIS, R.F.; FERREIRA, R.P.; NEVES, J.C.L. et al. Absorção de fósforo e crescimento de milho com sistema radicular parcialmente exposto a fonte de fósforo. Pesquisa Agropecuária Brasileira, v.20, p.749-754, 1985.

RUPPIN, R.F. Níveis críticos de fósforo no solo e na planta para o estabelecimento de capim-elefante (Pennisetum purpureum) cv. Napier. Viçosa, MG: Universidade Federal de 
Viçosa, 1997. 58p. Dissertação (Mestrado em Zootecnia) Universidade Federal de Viçosa, 1997.

SANTOS, H.Q.; FONSECA, D.M.; CANTARUTTI, R.B. et al. Níveis críticos de fósforo no solo e na planta para gramíneas forrageiras tropicais, em diferentes idades. Revista Brasileira de Ciência do Solo, v.26, p.173-182, 2002.

SARAIVA, O.F.; CARVALHO, M.M. Adubação nitrogenada e fosfatada para o estabelecimento de capim-elefante em latossolo vermelho-amarelo, textura argilosa. Revista Brasileira de Ciência do Solo, v.15, p.201-205, 1991.

SILVA, D.J; QUEIROZ, A.C. Análise de alimentos: métodos químicos e biológicos. 3.ed. Viçosa, MG: Universidade Federal de Viçosa, 2002. 235p.

THOMAS, G.W.; PEASLEE, D.E. Testing soils for phosphorus. In: WALSH, L.M.; BEATON, J.D. (Eds.) Soil testing and plant analysis. Madison: Soil Science Society American, 1973. p.115-129.

UNIVERSIDADE FEDERAL DE VIÇOSA - UFV. SAEG - Sistema de análises estatísticas e genéticas. Versão 5.0. Viçosa, MG: 1995. 150p. (Manual do usuário).
VALE, F.R. Efeito do alumínio sobre a cinética de absorção de nitrato, amônio e fosfato em milho (Zea mays, L.) e em clone de eucalipto (Eucalyptus alba). Viçosa, MG: Universidade Federal de Viçosa, 1982.71p. Dissertação (Mestrado em Solos e Nutrição de Plantas) - Universidade Federal de Viçosa, 1982.

VETTORI, L. Métodos de análise de solo. Rio de Janeiro: Ministério da Agricultura, 1969. 24p (Boletim Técnico, 7).

WERnER, J.C. Adubação de pastagens. 2.ed. Nova Odessa: Instituto de Zootecnia, 1986. 49p.

Recebido: 25/01/05 Aprovado: 19/12/05 